\title{
Productivité et utilisation de Calliandra calothyrsus pâturé par le cerf rusa en fonction de sa densité de plantation et de sa hauteur de recépage
}

\author{
C. Corniaux ${ }^{1 *}$ S. Le Bel ${ }^{1}$ J.M. Sarrailh ${ }^{2}$
}

\begin{abstract}
Mots-clés
Cervidae - Cervus timorensis russa Calliandra calothyrsus - Gestion Plantation - N ouvelle-Calédonie.
\end{abstract}

\begin{abstract}
Résumé
Réalisée en Nouvelle-Calédonie, cette étude montre que les meilleures productivités annuelles de Calliandra calothyrsus (provenance San Ramon) sont obtenues lorsqu'ils sont recépés trimestriellement à $1 \mathrm{~m}$ de hauteur pour une densité de plantation de 10000 pieds par hectare $(2 \mathrm{~m} \times 0,5 \mathrm{~m}$ ou $1 \mathrm{~m} \times 1 \mathrm{~m}$, avec respectivement 9,40 et 9,67 te matière sèche produite par hectare par an). $D$ ans ces conditions, les refus sur les arbustes pâturés par le cerf rusa sont les plus faibles (respectivement 1,15 et 1,26 t MS/ha/an) et, par conséquent, l'ingéré y est maximal (respectivement 8,25 et 8,41 t MS/ha/an). Compte tenu de son comportement alimentaire, I'association cerf rusa-Calliandra calothyrsus est particulièrement prometteuse. Cet arbuste pourrait ainsi se substituer efficacement au Leucaena leucocephala, parasité par le psylle, dans les élevages de cervidés.
\end{abstract}

\section{INTRODUCTION}

En Nouvelle-Calédonie, comme dans la plupart des pays de la zone Pacifique (Australie, Indonésie), les ressources fourragères ont été sensiblement réduites depuis 1986 à la suite de la régression importante des populations de Leucaena leucocephala parasitées par le psylle (Heteropsylla cubana) $(3,10,27)$. Les solutions envisagées ont associé la lutte biologique par l'utilisation d'insectes prédateurs (4) et le recours à d'autres arbustes résistants au psylle (3). Parmi ceux-ci figurent certaines espèces locales ou exotiques dont la nature et le mode d'exploitation sont actuellement à l'étude $(5,6)$. Dans ce cadre, Calliandra calothyrsus, comparé notamment avec Gliricidia sepium ou Acacia ampliceps, semble particulièrement prometteur (26).

Par ailleurs, l'élevage du cerf rusa (Cervus timorensis russa) s'est sensiblement développé en Nouvelle-Calédonie ces dix dernières années (13). Son alimentation, plus particulièrement la gestion de ressources fourragères adaptées à son comportement alimentaire, apparaît pourtant comme un facteur limitant pour cette production. En tant que consommateur d'herbacées et de fourrages aériens, son élevage justifie des efforts de diffusion d'arbustes fourragers pour les pâturages artificiels ou les parcours extensifs.

1. Cirad-élevage, Port-Laguerre, BP 186, 98848 Nouméa, Nouvelle-Calédonie 2. Cirad-forêt, Montravel, BP 186, 98848 Nouméa, Nouvelle-Calédonie * Adresse actuelle pour la correspondance : Isra/Cirad-emvt, BP 744, Saint-Louis, Sénégal
Dans ce contexte, un programme de recherche a été entrepris afin de connaître et de maîtriser l'utilisation par les cerfs rusa du Calliandra calothyrsus. L'un des principaux axes de travail a été de gérer l'exploitation des arbustes fourragers dans les conditions de pâturage. Cette étude présente la production de parcelles de Calliandra calothyrsus en fonction de leur densité de plantation et de leur hauteur de recépage. Des estimations des quantités ingérées par les cerfs y sont également présentées.

\section{MATERIEL ET METHODES}

\section{Site d'étude}

La Nouvelle-Calédonie est un archipel de $18575 \mathrm{~km}^{2}$ situé à proximité du Tropique du Capricorne entre 18 et $23^{\circ}$ de latitude $\mathrm{S}$. La température moyenne est comprise entre 22 et $24{ }^{\circ} \mathrm{C}$, allant d'un maximum en février à un minimum en juillet-août. Sur la façade Ouest de l'île, la moins arrosée, la pluviométrie varie de 800 à $1500 \mathrm{~mm}$ avec une saison des pluies (janvier à mars) et une saison sèche (septembre à décembre) relativement marquées. Durant cette étude (avril 1996-avril 1997), la pluviométrie relevée sur la station expérimentale de Port-Laguerre a été de $1178 \mathrm{~mm}$ relativement bien répartis tout au long de l'année, c'est-à-dire sans période sèche prolongée. La température moyenne s'est élevée à $22,3{ }^{\circ} \mathrm{C}$. L'essai a été établi sur des sols bruns (pente), qui présentaient de faibles réserves en eau, et sur des vertisols (bas de pente). 


\section{Dispositif}

La variété de Calliandra calothyrsus mise en place pour l'essai était San Ramon, originaire du Costa Rica, appréciée en NouvelleCalédonie pour sa productivité et son taux de germination élevé en pépinière (25). Les plants, non inoculés, ont été repiqués en janvier 1995 dans un carré mis en défens de 0,25 ha, au sein d'une parcelle d'un hectare au total. Dans chaque carré, quatre densités de plantation ont été appliquées :

- $2 \mathrm{~m} \times 2 \mathrm{~m}$, soit 2500 pieds/ha;

- $2 \mathrm{~m} \mathrm{x} 1 \mathrm{~m}$, soit 5000 pieds/ha;

- $2 \mathrm{~m} \times 0,5 \mathrm{~m}$, soit 10000 pieds/ha ;

- $1 \mathrm{~m} \mathrm{x} 1 \mathrm{~m}$, soit 10000 pieds/ha.

Un premier recépage a été effectué pour les quatre densités en janvier et février 1996, soit 13-14 mois après la plantation, selon trois niveaux :

- à 20-30 cm du sol, au gyrobroyeur ;

- à $50 \mathrm{~cm}$ du sol, au sabre d'abattis ;

- à $1 \mathrm{~m} \mathrm{du} \mathrm{sol,} \mathrm{au} \mathrm{sabre} \mathrm{d'abattis.}$

Quatre parcelles de ce type ont été utilisées pour l'essai dont trois pour les mesures. La quatrième servait de « tampon » afin de respecter les délais de rotation imposés par le protocole.

Entrés en avril 1996, soit trois mois après le recépage, une soixantaine de daguets ont été élevés sur ces parcelles conduites en rotation pendant une année (sortie en avril 1997). La charge annuelle a donc été de l'ordre de $450 \mathrm{~kg}$ PV/ha et la charge instantanée de $1800 \mathrm{~kg}$ PV/ha. Le rythme de retour dans une parcelle était fixé à trois mois, soit quatre passages dans une année. Chaque parcelle était ainsi occupée 20 à 25 jours à chaque retour des animaux. A la sortie des cerfs de la parcelle pâturée, les arbustes étaient recépés aux trois hauteurs.

\section{Mesures et calculs}

Le jour précédant l'entrée des daguets dans une parcelle, 10 arbustes étaient récoltés (feuilles et rameaux chlorophylliens) pour chacune des 12 modalités du plan expérimental (4 densités x 3 hauteurs de recépage). La matière fraîche et la matière sèche (MS) étaient alors mesurées sur les 120 échantillons. Leur hauteur et leur nombre de tiges principales (au départ du collet) ont également été systématiquement notés. A la sortie des animaux de la parcelle, 7 à 10 arbustes étaient recépés pour chacune des 12 modalités afin de mesurer les refus en matière fraîche et en matière sèche. Pour l'obtention de la matière sèche, les échantillons ont été placés $48 \mathrm{~h}$ dans une étuve à $70^{\circ} \mathrm{C}$.

A partir de ces mesures, les quantités de matière sèche produite par pied et à l'hectare ont été calculées en fonction notamment de la hauteur de recépage et de la densité de plantation. Les quantités de matière sèche ingérée par les cerfs ont été estimées par différence entre les productions à l'entrée des animaux et les refus à leur sortie de la parcelle. Par conséquent, l'ingestion a été sous-estimée en ne prenant pas en compte la croissance des arbustes pendant la période de pâture. Cette croissance a toutefois semblé limitée et l'objet du protocole était avant tout la hiérarchisation entre les différentes modalités de l'expérimentation. Les analyses statistiques ont été réalisées par analyses de variance sur le logiciel SPSS.
RESU LTATS

\section{Productivité des Calliandra calothyrsus}

Les résultats synthétiques figurent dans le tableau I. On peut noter que la hauteur des arbustes était plus sensible à la saison qu'à la densité de plantation. De la même façon, il faut souligner que le nombre de tiges par pied dépendait davantage de la densité de plantation que de la hauteur de recépage. Ainsi, celui-ci était significativement supérieur pour les densités de plantation $2 \mathrm{~m}$ x $2 \mathrm{~m}$ et $2 \mathrm{~m} \times 1 \mathrm{~m}$ comparativement aux densités de plantation $2 \mathrm{~m}$ x $0,5 \mathrm{~m}$ et $1 \mathrm{~m} \times 1 \mathrm{~m}$. Le gyrobroyage a toutefois permis d'augmenter le nombre de tiges par rapport au recépage à $1 \mathrm{~m}$ et à $50 \mathrm{~cm}$.

Les meilleures productions de matière sèche par pied ont été obtenues avec le recépage à $1 \mathrm{~m}$, avant celui à $50 \mathrm{~cm}$ et le gyrobroyage. Pour la densité de plantation, la hiérarchie a été dominée par $2 \mathrm{~m}$ x $2 \mathrm{~m}$. Les arbustes plantés à 2500 plants par hectare ont en effet bénéficié d'un espace vital plus important. En revanche, la hiérarchie était modifiée lorsque les rendements à l'hectare étaient considérés. Les meilleures performances étaient alors atteintes avec une hauteur de recépage à $1 \mathrm{~m}$ et des densités de plantation de 10000 pieds par hectare $(1 \mathrm{~m}$ x $1 \mathrm{~m}$ ou $2 \mathrm{~m}$ x $0,5 \mathrm{~m}$, respectivement 9,67 t MS/ha/an et 9,40 t MS/ha/an), nettement supérieures aux densités de 5000 et 2500 pieds par hectare (respectivement 6,29 t MS/ha/an et 5,47 t MS/ha/an).

Inversement, certaines densités de plantation étaient particulièrement dépréciées à des hauteurs de recépage données (effet densité $\mathrm{x}$ hauteur très significatif). Ainsi la production de matière sèche était sensiblement plus faible pour les densités $2 \mathrm{~m}$ x $0,5 \mathrm{~m}$ et $2 \mathrm{~m}$ x $1 \mathrm{~m}$ sur les carrés gyrobroyés.

Enfin, il faut signaler la forte variabilité intra-traitement et intraparcelle des productions de matière sèche comme en témoignent les écart-types relativement élevés.

\section{Utilisation par les cerfs rusa}

Le tableau II présente les refus de Calliandra calothyrsus pâturé par les cerfs ainsi que l'estimation de l'ingéré en fonction de la hauteur de recépage et de la densité de plantation. Comme pour les données de productivité, les effets « hauteur de recépage » et « densité de plantation » étaient significatifs pour l'ensemble des variables mesurées ou calculées. En revanche, l'effet « passage des animaux » n'était significatif $(\mathrm{P}<0,01)$ que pour les refus par pied alors que l'effet « parcelle» ne l'était $(\mathrm{P}<0,05)$ que pour l'ingéré par pied ou à l'hectare.

En fonction de la hauteur de recépage, on constate que les refus étaient d'autant plus faibles que la production en MS des arbustes était élevée. Les cerfs ont donc utilisé les arbustes de façon plus efficiente lorsqu'ils étaient coupés à $1 \mathrm{~m}$ de hauteur en raison notamment d'une production inférieure de bois ou de rameaux chlorophylliens qui constituaient l'essentiel des refus (ratio feuilles/tiges favorable et nombre de tiges plus faible). Par leur comportement, les cerfs semblaient également passer davantage de temps dans les carrés recépés à $1 \mathrm{~m}$ de hauteur où ils pouvaient facilement s'abriter.

En revanche, les parcelles les moins denses, où la production par pied était la plus élevée, avaient les refus les plus importants. Cela s'explique également par une production de bois et de rameaux chlorophylliens plus importante. 


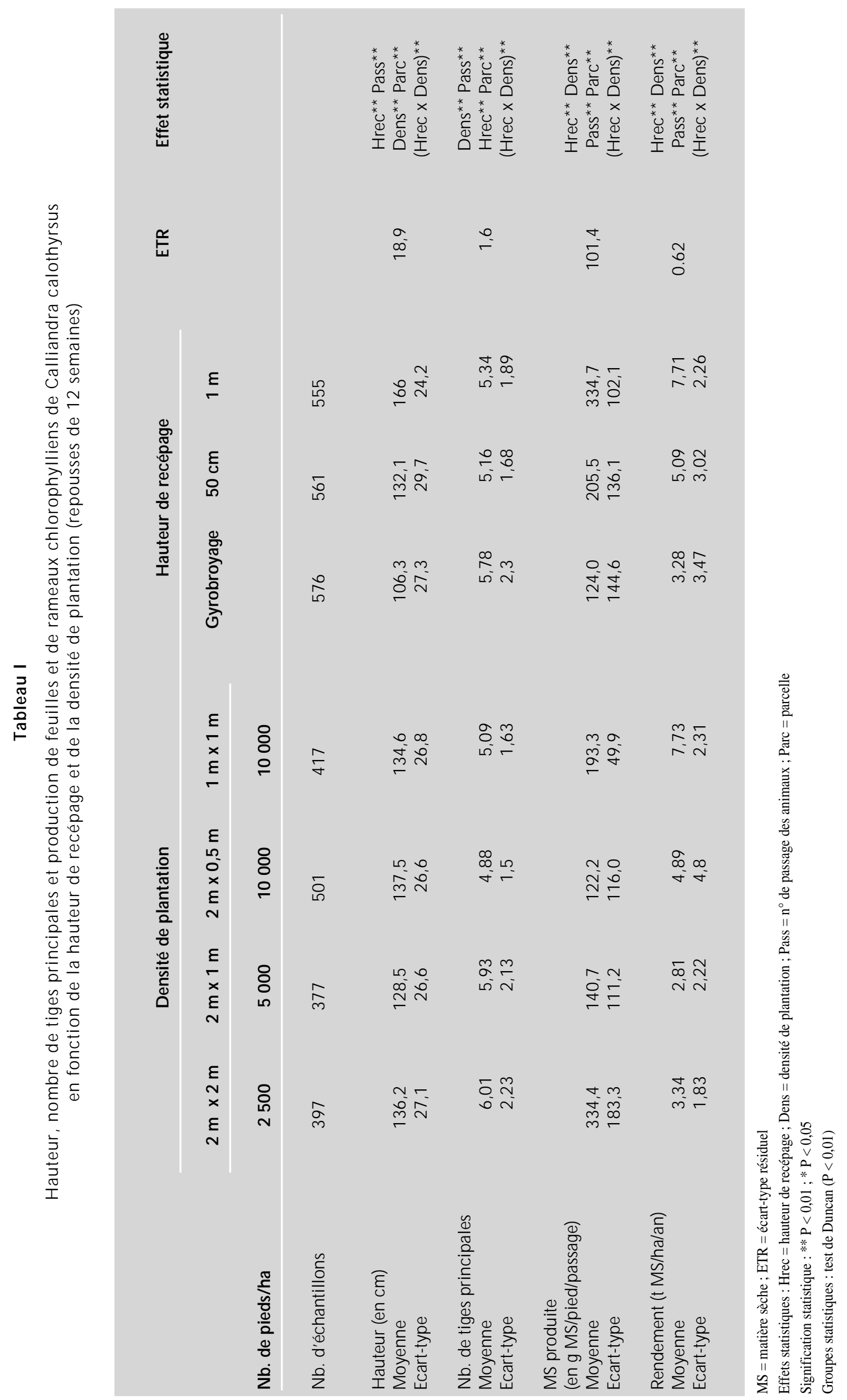


Management of Calliandra calothyrsus grazed by rusa deer

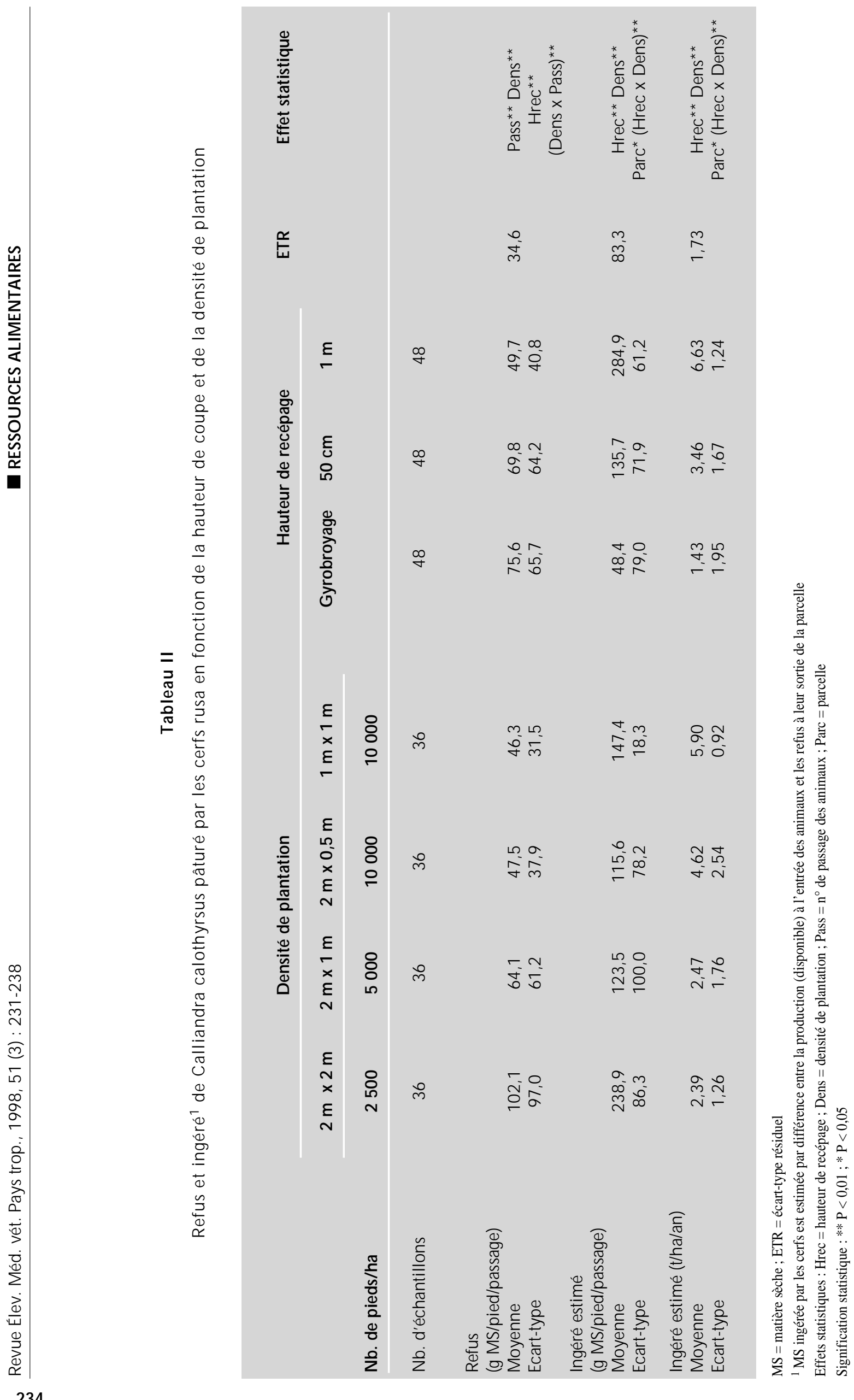


L'estimation de l'ingéré en tonnes par hectare par an a montré que, comme pour le rendement en MS, les meilleurs résultats ont été obtenus pour une hauteur de recépage à $1 \mathrm{~m}$ et des densités de plantation à 10000 pieds/ha (respectivement 8,25 et 8,41 t/ha pour $2 \mathrm{~m}$ x $0,5 \mathrm{~m}$ et $1 \mathrm{~m}$ x $1 \mathrm{~m}$ ). En outre, la différence avec les autres modalités a été renforcée par des valeurs de refus les plus faibles de l'essai (respectivement 28,8 et 31,7 g MS/pied). Un effet « hauteur de recépage $\mathrm{x}$ densité de plantation » sur l'ingéré très significatif $(\mathrm{P}<0,01)$ a également été constaté au profit de ces deux modalités.

\section{Effets du numéro de cycle de pâturage}

La production en MS et les quantités de refus ont été sensiblement supérieures pendant le premier trimestre de l'essai (correspondant au premier passage des animaux dans les parcelles). Ceci était lié à une pluviométrie conséquente par rapport aux trois autres trimestres qui a engendré une production de bois et de rameaux chlorophylliens plus importante, d'où une augmentation des quantités de MS produites et refusées.

Cependant, les variations des quantités de MS produites et ingérées n'étaient pas semblables selon la densité de plantation ou la hauteur de recépage. Ainsi, les figures 1 et 2 montrent une relative stabilité tout au long de l'année de la production de matière sèche par pied pour une hauteur de coupe à $1 \mathrm{~m}$ et une densité de 10000 pieds à l'hectare. En revanche, la production a chuté d'une manière quasi linéaire lorsque les arbustes ont été recépés par gyrobroyage (figure 2). Pour une hauteur de recépage à $1 \mathrm{~m}$ et une densité de plantation de $2 \mathrm{~m}$ x $0,5 \mathrm{~m}$ ou de $1 \mathrm{~m} \mathrm{x} 1 \mathrm{~m}$, la production et l'ingestion de Calliandra calothyrsus étaient non seulement élevées, comme cela a été souligné ci-dessus, mais elles étaient aussi relativement stables.

\section{DISCUSSION}

\section{Gestion de la plantation de Calliandra calothyrsus}

Gérer une plantation d'arbustes fourragers consiste à maximiser la production de matériel végétal consommable par les animaux tout en pérennisant la ressource. Les facteurs majeurs de cette gestion sont : la densité de plantation, la date de la première coupe (ou premier passage des animaux), la hauteur de coupe (ou de recépage) et la fréquence de coupe (ou de recépage).

\section{Densité de plantation}

Dans cette étude, les meilleures productivités de Calliandra calothyrsus ont été obtenues à la densité de 10000 pieds/ha $(2 \mathrm{~m}$ x $0,5 \mathrm{~m}$ ou $1 \mathrm{~m}$ x $1 \mathrm{~m}$ ). Ella et coll. (9) confirment la supériorité d'une plantation à cette densité par rapport à des densités inférieures. Néanmoins, ils obtiennent de meilleurs rendements à 20000 pieds/ha (1 m x 0,5 m). Cette densité est cependant conçue dans l'optique d'une production de bois et est peu compatible avec l'élevage de cerfs. En effet, les risques de casse des arbustes ou de blessures des animaux seraient amplifiés. De plus, la manipulation des cerfs est, pour ainsi dire, impossible avec cette densité.

Il faut par conséquent privilégier une densité à 10000 pieds/ha où la production de fourrage est maximale. L'éleveur devra faire un choix entre une plantation à $2 \mathrm{~m}$ x $0,5 \mathrm{~m}$ ou à $1 \mathrm{~m} \times 1 \mathrm{~m}$. La première présente l'avantage de faciliter la manipulation des animaux et le recépage grâce à son inter-rang relativement large. La seconde permet de limiter les adventices et d'être plus en harmonie avec le comportement des cerfs en raison du recouvrement quasi total de la parcelle par Calliandra.

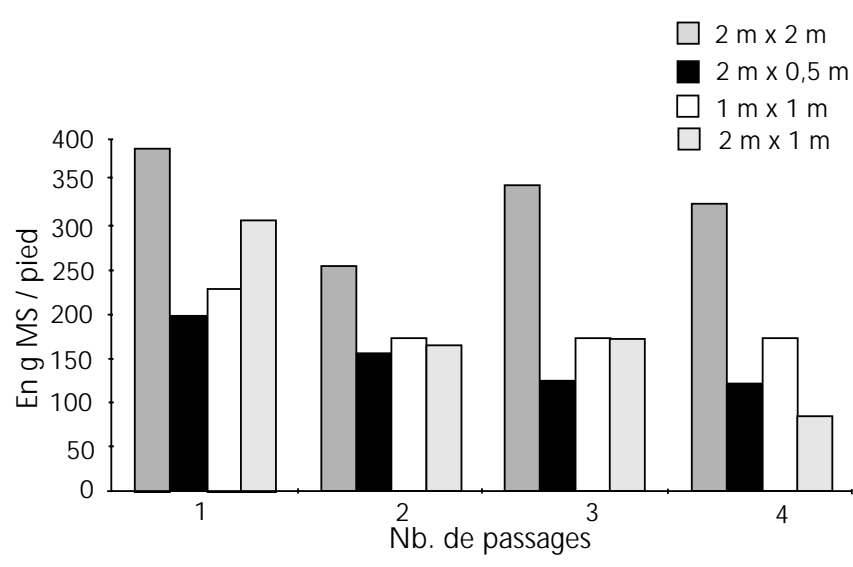

Figure 1 : production de MS/pied en fonction du $n^{\circ}$ de passage et de la densité de plantation.

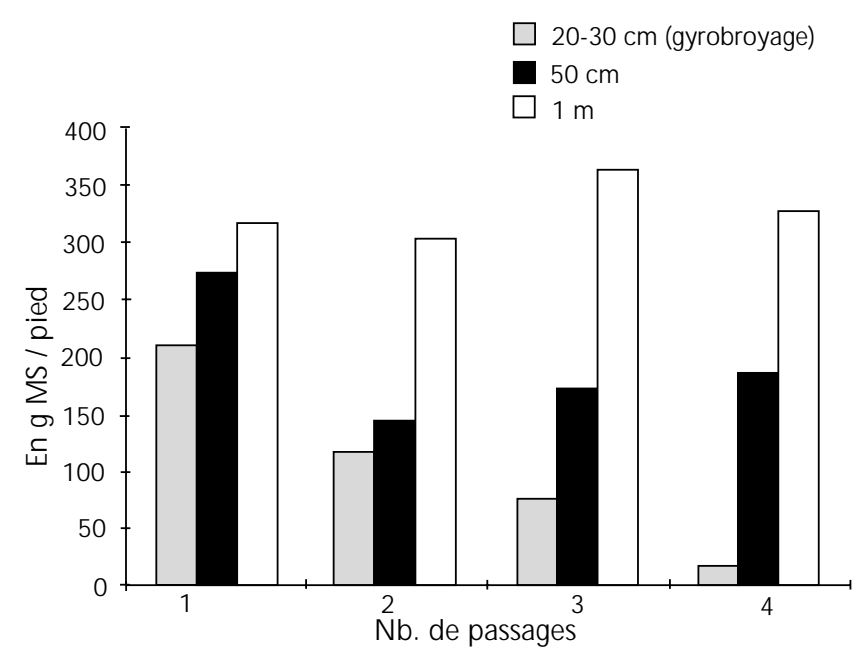

Figure 2 : production de MS/pied en fonction du $n^{\circ}$ de passage et de la hauteur de coupe.

\section{Date de la première coupe}

Tout en préservant sa productivité future, Calliandra calothyrsus est particulièrement précoce. Une première coupe est en effet possible à partir de 12 mois alors qu'elle est recommandée à 18 et 24 mois respectivement pour Leucaena leucocephala et Gliricidia sepium $(8,29)$. Aussi, le temps de mise en défens d'une parcelle plantée en Calliandra est réduit, ce qui est avantageux pour l'éleveur qui peut utiliser plus rapidement cette ressource fourragère. En Nouvelle-Calédonie, il est conseillé de planter cet arbuste fourrager en janvier au début de la saison des pluies pour l'exploiter 12 à 13 mois plus tard lors d'une nouvelle période pluvieuse (25).

\section{Hauteur de recépage}

L'utilisation du gyrobroyeur, très commune en Nouvelle-Calédonie, permet de réduire sensiblement la pénibilité du recépage. Bien que les résultats sur Leucaena leucocephala soient prometteurs, cette pratique engendre des difficultés de reprise voire de mortalité sur Calliandra calothyrsus (19). Dans la présente étude, il ressort que la productivité du Calliandra est encore plus affectée avec des densités de plantation de $2 \mathrm{~m}$ x $1 \mathrm{~m}$ et de $2 \mathrm{~m}$ x $0,5 \mathrm{~m}$. Une augmentation sensible des refus a également été constatée sur les carrés gyrobroyés parallèlement à un accroissement du nombre de tiges et de la production de bois ou de rameaux chlorophylliens. L'arbuste est en effet contraint, à cette hauteur de coupe, de refaire son architecture avant de produire des feuilles $(9,29)$. 
En appliquant un recépage à $50 \mathrm{~cm}$ de hauteur, les auteurs ont souhaité limiter les risques de mortalité par épluchage constatés sur des parcelles coupées à $1 \mathrm{~m}$ et pâturées par des chèvres (28). En fait, seuls quelques pieds avaient disparu sur les pentes à ces deux hauteurs de coupe mais probablement uniquement à cause de la sécheresse du terrain. En outre, les bris de branches et la mortalité n'étaient pas plus importants avec un élagage à $1 \mathrm{~m}$, à la différence de ce qui a été observé en élevage bovin (28).

La hauteur de coupe du Calliandra calothyrsus à $1 \mathrm{~m}$ est donc à privilégier pour un élevage de cerfs rusa. La productivité totale et consommable est alors maximale (tableau III). Compte tenu de la faiblesse des refus, le recépage est même facultatif : une remise à niveau annuelle semble tout à fait satisfaisante. Il faut enfin noter que le comportement de cet animal, relativement sauvage, favorise l'utilisation des arbustes à cette hauteur. Il aime en effet séjourner dans ces carrés où il peut facilement trouver un abri.

\section{Fréquence de coupe}

Les meilleures productivités des arbustes fourragers sont obtenues avec des fréquences de coupe de 12 à 16 semaines (29). En outre, ce rythme de défoliation permet de fournir une fraction consommable par les ruminants de l'ordre de 50 à 60 p. 100 du total produit. Dans un système de cut and carry, Calliandra calothyrsus semble plus productif avec une fréquence de coupe de 16 semaines (18). Roshetko et coll. (24) et Morikawa et coll. (16), dans des conditions de pluviométrie plus favorables, préconisent un rythme de 12 semaines.

En tenant compte de la production consommable, de la valeur alimentaire du Calliandra et de celle de la strate herbacée, une rotation tous les trimestres, soit 12 semaines, telle qu'elle a été appliquée dans cette étude, semble souhaitable. Pour les mêmes raisons, ce rythme est proposé par Mandret et Tassin à la Réunion (14). De plus, à cette fréquence de coupe, il faut noter que Calliandra calothyrsus est plus productif que Leucaena leucocephala et Gliricidia sepium (12; tableau III).

\section{Application à l'élevage du cerf rusa}

\section{Type d'élevage}

Compte tenu de ses exigences en eau, Calliandra calothyrsus (var. San Ramon) devra tout d'abord être mis en place dans des parcelles où la pluviométrie est supérieure à $1000 \mathrm{~mm} / \mathrm{an}$. En Nouvelle-Calédonie, cela correspond aux élevages du Sud, de la côte Est et du bas de chaîne sur la côte Ouest. D'autre part, en raison du coût élevé de la plantation, elle ne doit être envisagée que dans le cadre d'un système intensifié, notamment pour les ateliers d'embouche de daguets destinés au marché en vif ou en carcasse. Enfin, le pâturage doit être privilégié face au cut and carry. En effet, une fois coupées, l'appétabilité et la digestibilité des feuilles de Calliandra sont rapidement réduites en raison de leur forte teneur en tanins $(20,21)$.

\section{Un matériel végétal particulièrement adapté aux besoins du cerf rusa}

- Comportement du cerf rusa

Par son comportement alimentaire, le cerf rusa se nourrit naturellement d'arbustes fourragers. Par son caractère sauvage, il apprécie également les abris qu'ils lui fournissent. Aussi, Calliandra calothyrsus recépé à $1 \mathrm{~m}$ de hauteur et planté à une densité de 10000 pieds/ha est parfaitement adapté au comportement du cerf rusa. De plus, contrairement à d'autres ruminants (28), les daguets causent peu de mortalité sur Calliandra (moins de 3 p. 100 des sujets). Néanmoins, il est souhaitable d'éviter de faire pâturer ce type de parcelle par les mâles de plus de deux ans, sexuellement actifs, qui dégraderaient les arbustes avec leurs bois afin de marquer leur territoire en période de rut (13).

\section{- Valeur alimentaire du Calliandra}

L'appétabilité de Calliandra calothyrsus distribué en frais ou pâturé est médiocre à moyenne pour de nombreux ruminants $(2,22)$. En revanche, elle est élevée chez le cerf rusa, c'est-à-dire compa-

\section{Tableau III}

Productivité de Calliandra calothyrsus en fonction de sa densité de plantation et de sa hauteur de coupe

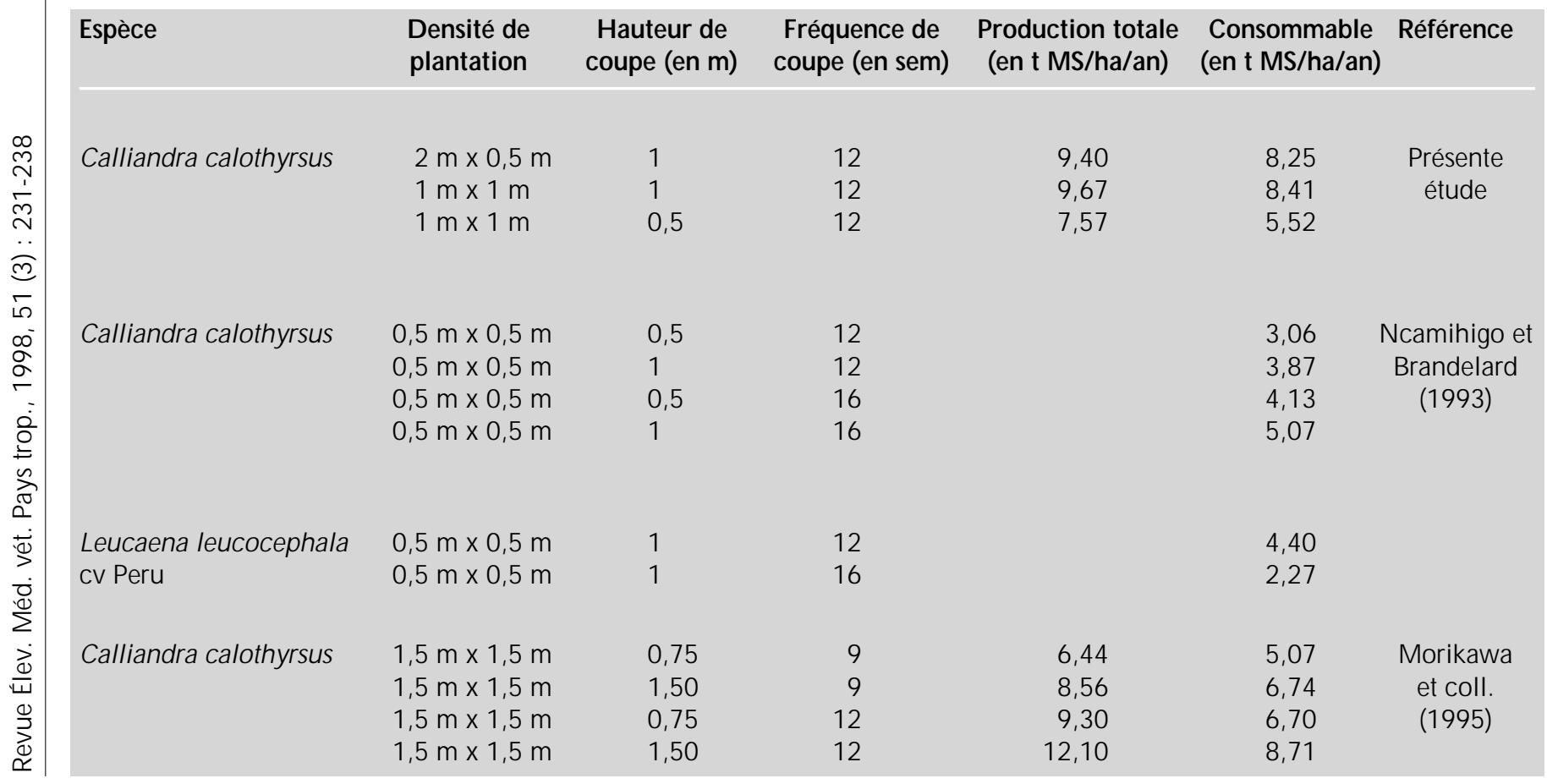


rable à celle de Leucaena leucocephala et supérieure à Gliricidia sepium (7). La présente étude montre également que l'ingéré est important sur des parcelles plantées à 10000 pieds/ha et recépées à $1 \mathrm{~m}$ de haut. Cet ingéré est de bonne qualité en raison de la richesse en matières azotées de cette légumineuse arbustive, de l'ordre de 20 à 25 p. $100 \mathrm{MS}$ (17). L'introduction de Calliandra calothyrsus à hauteur de 30 p. 100 dans la ration des ruminants permet, en outre, d'améliorer l'utilisation digestive des pâturages de qualité médiocre (22).

- Problème nutritionnel lié aux tanins...

Dans cette étude, les auteurs ont tenté de garder ces proportions en mettant en place une parcelle arbustive d'un quart d'hectare dans une parcelle herbacée d'un hectare et en appliquant une rotation trimestrielle. Or, ils ont observé que le cerf se nourrissait quasi exclusivement de Calliandra avant de pâturer la strate herbacée. Le régime alimentaire s'est alors trouvé déséquilibré et les refus sur la strate herbacée étaient importants. Cela devrait, a priori, conduire à limiter l'utilisation du Calliandra dans le cadre d'un élevage de cervidés. De plus, la teneur en tanins de la ration est particulièrement élevée lorsqu'elle n'est composée que de Calliandra. Ils peuvent déprécier sensiblement la valeur alimentaire du fourrage notamment chez les bovins et les moutons (15).

-... mais une dégradation digestive probablement adaptée chez le cerf rusa

La croissance des daguets, de l'ordre de $70 \mathrm{~g} / \mathrm{j}$ pour des animaux pesant plus de $35 \mathrm{~kg}$ de poids vif, n'est pas affectée (Le Bel, à paraître). Aussi, il est probable que, comme pour les chèvres férales (19), les protéines salivaires du cerf riches en proline (1) et sa flore ruminale adaptée (11) lui permettent de dégrader de façon efficiente les protéines alimentaires d'arbustes tel que Calliandra calothyrsus à teneur élevée en tanins. Les tanins pourraient aussi favoriser la formation de protéines by-pass, non dégradées dans le rumen et absorbées au niveau de l'intestin grêle (23).

\section{Perspectives}

Des expériences complémentaires doivent être entreprises avant que l'usage du Calliandra calothyrsus soit vulgarisable à grande échelle. Il serait intéressant de mesurer la productivité des parcelles lors des prochaines années afin de mieux apprécier leur longévité et de quantifier l'effet d'une répartition différente des pluies sur l'année. A l'avenir, il faudra aussi mettre au point une technique de semis direct du Calliandra calothyrsus pour pouvoir le diffuser chez les éleveurs. Actuellement, le coût élevé du repiquage après passage en pépinière rend la technique difficilement applicable sur le terrain. Par ailleurs, à la suite de cette étude, une expérimentation a été mise en place en 1997 sur la station de recherche de Port-Laguerre afin de mesurer les performances zootechniques des daguets nourris ou non avec du Calliandra. Ces travaux permettront également de préciser le chargement optimal à appliquer dans ce type de système fourrager. Enfin, il parait nécessaire d'étudier le rôle des tanins dans l'utilisation digestive de Calliandra calothyrsus par le cerf rusa.

\section{- CONCLUSION}

Beaucoup de travaux ont été menés en milieu tropical et subtropical afin de maximiser la productivité des arbustes fourragers tels que Leucaena leucocephala, Gliricidia sepium ou Calliandra calothyrsus. Pourtant, peu d'essais ont été conduits en conditions de pâturage, notamment pour ce dernier, et les références au cerf rusa sont rares. L'originalité de ce travail a consisté à apprécier, en Nouvelle-Calédonie, l'utilisation du Calliandra par le cerf rusa.
Les résultats expérimentaux montrent que les meilleures productivités de Calliandra calothyrsus sont obtenues lorsqu'ils sont recépés trimestriellement à $1 \mathrm{~m}$ de hauteur pour une densité de plantation de 10000 pieds/ha ( $2 \mathrm{~m} \mathrm{x} \mathrm{0,5} \mathrm{m} \mathrm{ou} 1 \mathrm{~m}$ x $1 \mathrm{~m})$. Dans ces conditions, les refus sur les arbustes pâturés par le cerf rusa sont les plus faibles et, par conséquent, l'ingéré (t MS/ha/an) y est maximal. Pratiquement, en matière de gestion du Calliandra, les conseils aux éleveurs devront encourager :

- une densité de plantation de 10000 pieds/ha ;

- un recépage annuel des arbustes à $1 \mathrm{~m}$ de hauteur ;

- une rotation trimestrielle sur les parcelles.

Contrairement aux autres ruminants, le cerf rusa valorise particulièrement bien Calliandra calothyrsus. Cet arbuste semble pouvoir remplacer efficacement Leucaena leucocephala, parasité par le psylle, notamment pour des ateliers d'embouche de daguets. Des études complémentaires devront toutefois être menées sur l'association très prometteuse constituée par Calliandra et cerf rusa. Outre les facteurs techniques (agronomiques et zootechniques), elles devront intégrer la rentabilité économique de cette association. Ce n'est qu'à ce niveau que l'éleveur adoptera ou non cette innovation dans son système d'exploitation.

\section{BIBLIO GRAPHIE}

1. AUSTIN P.J., SU CAHR L.A., ROBBINS C.T., HAGERMAN A.E, 1989. Tannin-binding proteins in saliva of deer and their absence in saliva of sheep and cattle. J. Chem. Ecol., 15: 1335-1347.

2. BERHE K., MOHAMED-SALEEM M.A., 1996. The potential of Calliandra calothyrsus as a fodder tree on acidic nitosols of the Southern, Western and Southwestern Highlands of Ethiopia. In: Evans D.O. Ed., Proc. int. Workshop on the genus Calliandra, Bogor, Indonesia, 23-27 January 1996. Morrilton, USA, Winrock International, p. 234-244.

3. BRAY R.A., 1994. The Leucaena psyllid. In: Gutteridge R.C., Shelton H.M. Eds., Forage tree legumes in tropical agriculture. Wallingford, UK, CAB International, $389 p$.

4. CHAZEAU J., CAPART I., BONNET de LARBOGNE L., 1992. Amélioration de l'état phytosanitaire du faux-mimosa Leucaena leucocephala en Nouvelle-Calédonie par le renforcement du complexe des ennemis naturels du psylle ravageur Heteropsylla cubana. N ouméa, Nouvelle-Calédonie, O rstom, 24 p. (Sciences de la Vie, Zoologie appliquée, Convention $\mathrm{n}^{\circ}$ 6)

5. CIRAD-EMVT, 1996. In : Journées techniques arbustes fourragers, Port-Laguerre, N ouvelle-Calédonie, 3-4 octobre 1996, 40 p.

6. CORNIAUX C., DURAND N., SARRAIHL J.M., GUERIN H., 1996. Composition chimique et dégradabilité enzymatique et in vitro d'espèces ligneuses arbustives utilisables par les ruminants dans les parcours extensifs de Nouvelle-Calédonie. 1. Typologie. Revue Elev. Méd. vét. Pays trop., $49: 60-67$.

7. CORNIAUX C., LE BEL S., SARRAILH J.M., 1997. Shrub palatibility to rusa deer (Cervus timorensis russa) in New Caledonia. Nouméa, Nouvelle-Calédonie, Cirad-Elevage, $5 \mathrm{p}$.

8. ELLA A., BLAIR G.J., STÜR W.W., 1991. Effect of age of forage tree legumes at the first cutting on subsequent production. Trop. Grassl., $\mathbf{2 5}$ 275-280.

9. ELLA A., JACO BSEN C., STÜR W.W., BLAIR G.J., 1989. Effect of plant density and cutting frequency on the productivity of four tree legumes. Trop. Grassl., 23: 28-34.

10. GUTTERIDGE R.C., SHELTON H.M., 1994. The role of forage tree legumes in cropping and grazing systems. In: Gutteridge R.C., Shelton H.M. Eds., Forage tree legumes in tropical agriculture. Wallingford, UK, $C A B$ International, $389 p$.

11. HOFFMAN R.R., 1985. Digestive physiology of deer. Their morphophysiological specialisation and adaptation. In: Fenessy P.F. Drew K.R. Eds., Biology of deer production, Proc. int. Conf., Dunedin, New Zealand, 13-18 February 1983. R. Soc. N.Z., 22: 393-408. 
12. KRECIK S.G., LANTAGNE D.O., GOLD M.A., ROSHETKO J.M. 1993. Cutting management of Leucaena leucocephala, Calliandra calothyrsus and Gliricidia sepium for fodder production. Nitrogen Fixing Tree Res. Rep., 11: 18-20.

13. LE BEL S., 1993. L'élevage du cerf rusa en Nouvelle-Calédonie : bilan et perspectives. Nouméa, Nouvelle-Calédonie, Cirad-Elevage, $30 \mathrm{p}$.

14. MANDRET G., TASSIN J., 1996. Intérêt fourrager de Calliandra calothyrsus en altitude à la Réunion. Revue Elev. Méd. vét. Pays trop., 49 : 335-339.

15. MERKEL R., POND K.R., BURNS J.C., FISHER D.S., 1996 Condensed tannins in Calliandra calothyrsus and their effects on feeding value. In: Evans D.0. Ed., Proc. int. W orkshop on the genus Calliandra, Bogor, Indonesia, 23-27 January 1996. Morrilton, USA, Winrock International, p. 222-233.

16. MORIKAWA R.T., LANTAGNE D.O., GOLD M.A., KRECIK S.G. ROSHETKO J.M., 1995. Management of Calliandra calothyrsus for fodder production in Jamaïca. Trop. Grassl., 29: 236-240.

17. NORTON B.W., 1994. The nutritive value of tree legumes. In Gutteridge R.C., Shelton H.M. Eds., Forage tree legumes in tropical agriculture. Wallingford, UK, CAB International, $389 \mathrm{p}$.

18. NCAMIHIGO O., BRANDELARD P., 1993. Effects of cutting heights and cutting frequencies on the annual yield of Leucaena and Calliandra hedges. In: Proc. 17th int. Grassland Congress, New Zealand and Q ueensland, Australia, 8-21 February 1993, p. 877-878.

19. PALMER B., IBRAHIM T.M., 1996. Calliandra calothyrsus forage for the tropics - a current assessment. In: Evans D.0. Ed., Proc. int Workshop on the genus Calliandra, Bogor, Indonesia, 23-27 January 1996. Morrilton, USA, W inrock International, p. 183-194.

20. PALM ER B., MACQ UEEN D.J., GUTTERIDGE R.C., 1994. Calliandra calothyrsus - a multipurpose tree legume for humid locations. In Gutteridge R.C., Shelton H.M. Eds., Forage tree legumes in tropical agriculture. Wallingford, UK, CAB International, $389 \mathrm{p}$.

21. PALM ER B., SCHLINK A.C., 1992. The effect of drying on the intake and rate of digestion of the shrub legume Calliandra calothyrsus. Trop. Grassl., 26: 89-93.

\section{Summary}

Corniaux C., Le Bel S., Sarrailh J.M. Production and use of Calliandra calothyrsus by rusa deer based on plant density and cutting height

This study, carried out in New Caledonia, showed that the best annual production of Calliandra calothyrsus, origin San Ramon, was obtained with quarterly cutting heights of $1 \mathrm{~m}$ and a density of 10,000 plants per hectare $(2 \mathrm{~m} \times 0.5 \mathrm{~m}$ or $1 \mathrm{~m} \times 1 \mathrm{~m}$, yielding 9.40 and $9.67 \mathrm{t}$ of dry matter per hectare per year, respectively). The parts of shrubs ungrazed by rusa deer were then minimum (1.15 and $1.26 \mathrm{t} \mathrm{DM} / \mathrm{ha} / \mathrm{year}$ ) and feed intake maximum (8.25 and $8.41 \mathrm{t} \mathrm{DM} / \mathrm{ha} / \mathrm{year}$, respectively). Given the browsing behavior of rusa deer its association with Calliandra calothyrsus is promising. In deer farms, the shrub could be an efficient substitute for Leucaena leucocephala, parasitized by psyllid.

Key words: Cervidae - Cervus timorensis russa - Calliandra calothyrsus - M anagement - Planting - N ew Caledonia.
22. PATERSON R.T., ROOTHAERT R.L., NYATTA O.Z., AKEYEAMPONG E., 1996. Experience with Calliandra calothyrsus as a feed for livestock in Africa. In: Evans D.0. Ed., Proc. int. Workshop on the genus Calliandra, Bogor, Indonesia, 23-27 January 1996. Morrilton, USA, Winrock International, p. 168-179.

23. PEREZ-MOLDONADO R.A., 1994. The chemical nature and biological activity of tannins in forage legumes fed to sheep and goats. Ph.D. Thesis, University of Queensland, Department of Agriculture, Australia.

24. ROSHETKO J.M., LANTAGNE D.O., GOLD M.A., MORIKAWA B., 1996. Recommendations for establishing and managing Calliandra calothyrsus as a fodder resource in Jamaïca. In: Evans D.0. Ed., Proc. int. Workshop on the genus Calliandra, Bogor, Indonesia, 23-27 January 1996. Morrilton, USA, W inrock International, p. 168-179.

25. SARRAILH J.M., CIVALLERO D., 1997. Résultats obtenus sur I'essai provenances Calliandra calothyrsus un an après la plantation. Nouméa, N ouvelle-Calédonie, Cirad-Forêt, 18 p.

26. SARRAILH J.M., CORNIAUX C., DESVALS L., LE BEL S., 1996. Calliandra, a panacea for N ew Caledonia? In: Evans D.0. Ed., Proc. int. Workshop on the genus Calliandra, Bogor, Indonesia, 23-27 January 1996. Morrilton, USA, Winrock International, p. 115-119.

27. SHELTON H.M., BREW BAKER J.L., 1994. Leucaena leucocephala the most widely used tree legume. In: Gutteridge R.C., Shelton H.M. Eds., Forage tree legumes in tropical agriculture. Wallingford, UK, CAB International, $389 \mathrm{p}$.

28. SHELTON H.M., NORTON B.W., MULLEN B.F., GUTTERIDGE R.C., 1996. Utilization and nutritive value of Calliandra calothyrsus for forage : a review of research at the University of Q ueensland. In: Evans D.O. Ed., Proc. int. Workshop on the genus Calliandra, Bogor, Indonesia, 23-27 January 1996. Morrilton, USA, W inrock International, p. $210-221$.

29. STÜR W.W., SHELTO N H.M., GUTTERID GE R.C., 1994. Defoliation management of forage tree legumes. In: Gutteridge R.C., Shelton H.M. Eds., Forage tree legumes in tropical agriculture. Wallingford, UK, CAB International, $389 \mathrm{p}$.

Reçu le 24.11.97, accepté le 6.11 .98

\section{Resumen}

Corniaux C., Le Bel S., Sarrailh J.M. Productividad y uso del pasto Calliandra calothyrsus para el ciervo rusa, en función de su densidad de plantación y de su altura de poda

Este estudio, llevado a cabo en Nueva Caledonia, demuestra que las mejores productividades anuales de Calliandra calothyrsus (proveniente de San Ramon) se obtienen con podas trimestrales a $1 \mathrm{~m}$ de altura, para una densidad de plantación de 10000 pies por hectárea $(2 \mathrm{~m} \times 0,5 \mathrm{mo} 1 \mathrm{~m} \times 1 \mathrm{~m}$, con 9,40 y 9,67 te materia seca producida por hectárea por año, respectivamente). En estas condiciones, el rechazo de pastoreo de los arbustos por el ciervo rusa es mínimo $(1,15$ y $1,26 \mathrm{t}$ MS/ha/año, respectivamente) y por lo consiguiente, la ingestión es máxima (8,25 y 8,41 t M S/ha/año, respectivamente). Tomando en cuenta el comportamiento alimenticio del ciervo rusa, su asociación con Calliandra calothyrsus es particularmente prometedora. Este arbusto podría entonces substituir eficazmente a Leucaena leucocephala, parasitada por el silo, en los criaderos de ciervos.

Palabras clave: Cervidae - Cervus timorensis russa - Calliandra calothyrsus - G estión - Plantación - Nueva Caledonia. 